\title{
Increasing the Astrophysical Reach of the Advanced Virgo Detector via the Application of Squeezed Vacuum States of Light
}

\author{
F. Acernese et al. \\ (Virgo Collaboration) \\ Henning Vahlbruch, Moritz Mehmet, Harald Lück, and Karsten Danzmann \\ Institut für Gravitationsphysik, Leibniz Universität Hannover and Max-Planck-Institut für Gravitationsphysik (Albert-Einstein-Institut), \\ Callinstr. 38, 30167 Hannover, Germany
}

(Received 11 November 2019; published 5 December 2019)

\begin{abstract}
Current interferometric gravitational-wave detectors are limited by quantum noise over a wide range of their measurement bandwidth. One method to overcome the quantum limit is the injection of squeezed vacuum states of light into the interferometer's dark port. Here, we report on the successful application of this quantum technology to improve the shot noise limited sensitivity of the Advanced Virgo gravitationalwave detector. A sensitivity enhancement of up to $3.2 \pm 0.1 \mathrm{~dB}$ beyond the shot noise limit is achieved. This nonclassical improvement corresponds to a 5\%-8\% increase of the binary neutron star horizon. The squeezing injection was fully automated and over the first 5 months of the third joint LIGO-Virgo observation run O3 squeezing was applied for more than $99 \%$ of the science time. During this period several gravitational-wave candidates have been recorded.
\end{abstract}

DOI: $10.1103 /$ PhysRevLett.123.231108

\section{INTRODUCTION}

The current operating ground-based gravitational-wave (GW) detectors, namely Advanced LIGO [1], Advanced Virgo [2], and GEO600 [3], are based on kilometer-scale Michelson-type laser interferometers. The advanced observatories are among the most sensitive instruments nowadays and can achieve strain sensitivities at a level around $10^{-23} / \sqrt{\mathrm{Hz}}$ in the audio band. Because of their effective decoupling from environmental and technical noise sources, these detectors are limited in their sensitivity at most frequencies by quantum noise, which arises from the quantum nature of light and is driven by vacuum fluctuations of the optical field entering from the dark port of the interferometer. Quantum light fluctuations affect the Advanced Virgo detector via two uncorrelated mechanisms: the radiation pressure noise that is directly proportional to the optical power impinging on the test masses and inversely proportional to the square of the Fourier frequency, and the shot noise that is inversely proportional to the operating optical power. The fact that quantum shot noise and quantum radiation-pressure noise have an inverse dependence on power and that the underlying vacuum fluctuations of phase

*Full author list given at the end of the Letter.

Published by the American Physical Society under the terms of the Creative Commons Attribution 4.0 International license. Further distribution of this work must maintain attribution to the author(s) and the published article's title, journal citation, and DOI. and amplitude are uncorrelated gives rise to a so-called standard quantum limit (SQL) for continuous high-precision interferometric measurements [4,5].

A key technology to improve the GW detector sensitivity beyond this quantum noise limit is the injection of squeezed vacuum states of light into the dark port of the interferometer [6]. Since up to now the quantum radiation pressure noise is just below the residual technical noise sources at low GW detection frequencies, a broadband sensitivity improvement can be achieved by reducing the shot noise contribution via a moderate injection of frequencyindependent squeezed vacuum states, whose fluctuations are reduced in the quadrature of the light aligned with the gravitational-wave signal. In this case, the effect of squeezing injection is equivalent to an increase of the circulating optical power in the detector, without the drawback of an increase of the thermal effects inside the interferometer optics due to their residual absorption and consequent thermal aberrations [7]. In general the amount of quantum noise reduction due to the squeezing technology is limited by optical loss and phase noise [8,9]. Since the first observation of squeezed light in 1985 [10], squeezed light sources have constantly been improved, recently reaching a squeeze factor of up to $14 \mathrm{~dB}$ available for the injection into a GW detector [11]. The GEO600 detector, where squeezing enhancement is routinely employed since 2010 [12,13], has recently demonstrated a quantum noise reduction of $6 \mathrm{~dB}[14,15]$. About $2 \mathrm{~dB}$ quantum noise reduction has been also demonstrated in a proof-of-principle experiment in the initial LIGO detector [16]. Finally, during the 
current scientific run, the LIGO detector is operating in a stable and continuos way with up to $3 \mathrm{~dB}$ of squeezing [17].

Here, we report on the demonstration of a substantial and continuous reduction of the quantum shot noise in the Advanced Virgo detector via the injection of squeezed vacuum states of light. Squeezed states are generated on an external optical bench and subsequently injected into the interferometer. A quantum enhancement of up to $3.2 \pm 0.1 \mathrm{~dB}$ was measured at frequencies between $100 \mathrm{~Hz}$ and $3.2 \mathrm{kHz}$. The implementation of the squeezing technique was part of the Advanced Virgo upgrade program and has been operated with a $99 \%$ duty cycle of the recorded science data during the ongoing third joint LIGOVirgo observation run $\mathrm{O} 3$.

\section{EXPERIMENTAL SETUP}

Figure 1 shows a simplified optical layout of the Advanced Virgo (AdV) detector and the squeezed light generation. Advanced Virgo is a power-recycled Michelson interferometer with $3 \mathrm{~km}$ long Fabry-Perot cavities in the two orthogonal arms. The input beam, generated from a continuous wave laser source at a wavelength of $1064 \mathrm{~nm}$, has an optical power of $18 \mathrm{~W}$ and passes through a mode cleaning ring cavity for modal and frequency filtering before entering the interferometer. The circulating optical power is enhanced by a power recycling cavity, which is about $12 \mathrm{~m}$ long and is formed by placing an additional partially reflecting mirror (power recycling mirror) between the laser and the Fabry-Perot Michelson interferoemter. The gravitational-wave signal is sensed at the antisymmetric

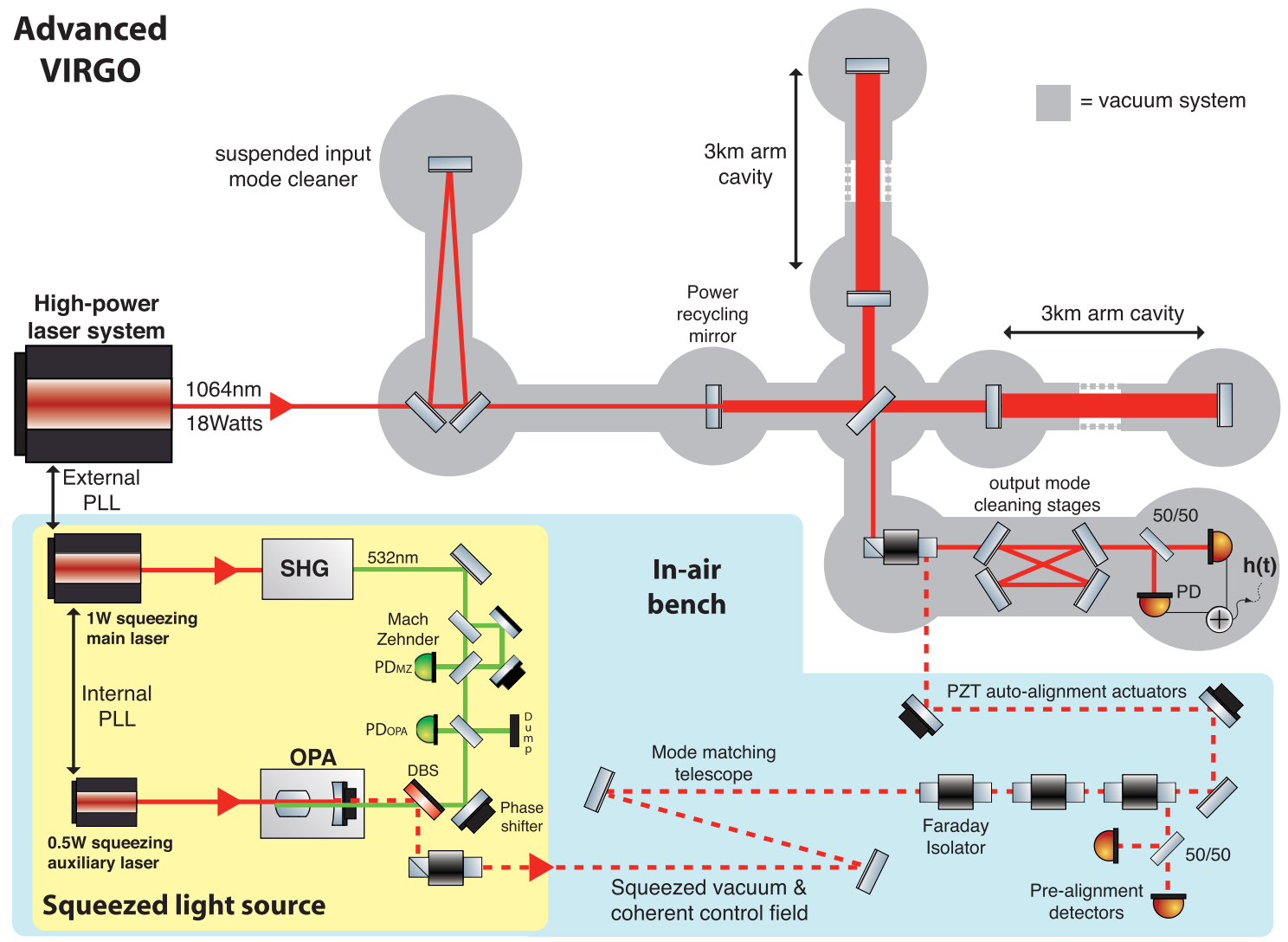

FIG. 1. Simplified layout of the quantum enhanced Advanced Virgo gravitational-wave detector. The Advanced Virgo detector is a power recycled Michelson interferometer using $3 \mathrm{~km}$ long Fabry-Perot cavities in the arms. Before being injected into the interferometer, the high power $(18 \mathrm{~W})$ input laser beam passes through a $143 \mathrm{~m}$ long triangular mode cleaner cavity. After the recombination at the central beam splitter, a fraction of the carrier light copropagates with the signal field to be used for a dc readout scheme. This output field is spatially filtered by an output mode cleaning stage and divided into two beams before detection. The sum of the two photo detectors is used to derive the gravitational-wave signal $h(t)$. All the interferometer optics as well as the injection and the detection benches are suspended and operated in vacuum. Squeezed vacuum states of light are prepared externally on an optical bench (blue box). This in-air bench hosts the squeezed light source (yellow box), the reflective mode matching telescope, the Faraday isolators, and alignment steering mirrors. The bench is covered with an enclosure to protect the optics against acoustic noise and air turbulence and is passively suspended by means of elastomer attenuators. More sophisticated suspension stages are not required as below $\sim 30 \mathrm{~Hz}$ the squeezer backscattered light effect is dominated by the up-conversion of the low frequency $(\sim 0.15 \mathrm{~Hz})$ microseismic peak [18,19]. Finally, the squeezed beam is injected into the interferometer vacuum system through an antireflectively coated viewport. The path between the acoustic enclosure and this optical window is shielded by a tube from air turbulence. 
port of the interferometer using a dc readout technique [20] after filtering by two cascaded, monolithic bow-tie optical cavities called output mode cleaners [21]. The light transmitted through these output mode cleaners (OMCs) is detected on a pair of photodiodes placed in the output ports of a 50\% beam splitter. The photo diode signals are digitally sampled from dc up to $\sim 400 \mathrm{MHz}$. The interferometer strain readout is derived from the sum of the signals in the audio band, while digital demodulation of rf sidebands provides several control signals. Furthermore, the use of two detectors allows one to identify possible technical noise sources through correlation measurements [22]. To isolate the readout system from environmental noise sources, the output mode matching optics, the OMCs, and the photo detectors for GW sensing are also suspended within the vacuum system. A more detailed description of the AdV detector can be found in [23].

An in-air optical table located close to the vacuum chambers containing the readout system hosts the squeezed light source itself and the optics required to inject the squeezed light beam into the dark port of the interferometer. The squeezed light source (yellow box in Fig. 1) is a standalone system constructed on a $1 \mathrm{~m}^{2}$ breadboard. The squeezer main laser is locked to the frequency of the Virgo detector's high power laser system with an external phase locked loop (PLL). The squeezer auxiliary laser is $7 \mathrm{MHz}$ frequency offset locked to the main squeezer laser via an internal PLL. Both squeezer lasers are single-frequency, continuous-wave nonplanar ring oscillators. A fraction of the squeezer main laser is sent into a second-harmonic generator (SHG) to provide a $532 \mathrm{~nm}$ pump field to drive an optical parametric amplifier (OPA). An electronically controlled, compact Mach-Zehnder interferometer is inserted in the green path for pump power stabilization and power control for adjustment of the parametric amplification factor inside the OPA. A detailed description of the SHG and the electronic controls can be found in [11]. The OPA is realized as a linear, doubly resonant cavity consisting of a piezoactuated coupling mirror and a periodically poled potassium titanyl phosphate (PPKTP) crystal. To simultaneously ensure phase matching and coresonance of both wavelengths, the nonlinear crystal is temperature stabilized to $\mathrm{mK}$ precision. The cavity length is controlled via a Pound-Drever-Hall locking scheme sensing a fraction of the green pump field reflected off the OPA. The generated infrared squeezed vacuum field exiting the OPA is separated from the green pump field via a dichroic mirror. A Faraday isolator on the squeezer breadboard serves as a first isolation stage to protect the OPA from back-reflected light which is known to introduce excess noise at audio-band Fourier frequencies due to parasitic interferences [24-26]. The frequency shifted auxiliary laser field is used to generate a coherent control field [27]. This dim field (approximately $1 \mu \mathrm{W}$ ) copropagates with the squeezed vacuum field and is used to control both the OPA pump phase and the squeezing phase.
A catoptric telescope close to the squeezer breadboard is used to mode match the squeezer output to the interferometer readout mode. The telescope is made of two off-axis spherical mirrors in order to compensate for residual astigmatism in the interferometer beam. Fine-tuning of the beam waist size and position is possible via micrometer stages on which the telescope mirrors are mounted. A triplet of low-loss Faraday isolators, with more than $40 \mathrm{~dB}$ isolation and less than 1\% single-pass loss each [28], serves as another high performance optical diode to suppress backscattering from light leaking out of the interferometer $[18,29]$. A pair of piezo-actuated tip-tilt steering mirrors with a separation of $\sim 1 \mathrm{~m}$ is used for initial tuning and serves as the actuator for automatic alignment of the squeezed light into the interferometer. The beam enters the vacuum system hosting the suspended detection benches through a wedged optical window and is injected into the interferometer through the output Brewster polarizer of a Faraday isolator, similar to the ones on the external table.

\section{AUTOMATION AND CONTROLS}

The automation and control of squeezed light injection is managed by a finite state machine based on the Metatron environment [30]. The engagement of the entire squeezing subsystem includes the remote control of the two PLLs, the squeezed light source, the coherent control loop, and the squeezing automatic alignment (AA). All these control loops are closed in parallel to the interferometer lock acquisition sequence, except for the coherent control loop, which is closed about 15-30 seconds after the interferometer has acquired complete lock.

\section{A. Phase-locked loops}

The frequency of the squeezer main laser is synchronized to the carrier frequency of the Advanced Virgo laser by the external PLL. For this a pick-off from the high power laser is $80 \mathrm{MHz}$ frequency shifted with an acousto-optic modulator $(\mathrm{AOM})$ and sent through a $54 \mathrm{~m}$ long polarization maintaining optical fiber onto the in-air bench. The frequency offset of the external PLL is controlled to exactly compensate the $80 \mathrm{MHz}$ frequency shift. The PLL between the two squeezer lasers (internal PLL) is operated with a $7 \mathrm{MHz}$ frequency offset. Both loops use feedback to the lasers' frequency actuators with a control bandwidth of $40-45 \mathrm{kHz}$.

\section{B. Squeezed light source}

The squeezed light source locking sequence is initiated after the PLLs have acquired lock. The Metatron remote controls four analog control loops, which are used to stabilize the SHG and OPA cavity lengths, the working point of the Mach-Zehnder interferometer, and the phase of the green OPA pump field. The bandwidth of these locking loops is about $10 \mathrm{kHz}$. 


\section{Coherent control loop}

The squeezing ellipse angle $\theta$ is directly related to the phase $\phi_{c c}$ of the $7 \mathrm{MHz}$ coherent control field [27]. Thus the value of $\theta$ can be stabilized by keeping $\phi_{c c}$ constant in time. The interference of the interferometer carrier field with the coherent control field generates beat notes at $\pm 7 \mathrm{MHz}$ on the detection photodiodes. The signal of one photodiode is digitally demodulated to infer the value of $\phi_{c c}$. The derived correction signal is converted into an analog feedback for actuating on the frequency modulation input of the $\mathrm{rf}$ generator employed for the external $80 \mathrm{MHz}$ PLL. The bandwidth of this control loop is about $5.5 \mathrm{kHz}$. The working point of the loop is stabilized against low frequency drifts using a variant of the method described in Ref. [13]. In our case a dither line at $2.8 \mathrm{kHz}$ is superimposed to the coherent control loop correction signal producing a signal at the same frequency in the demodulated amplitude of the $7 \mathrm{MHz}$ beat note. It can be shown that the amplitude of this signal is directly linked to the angle of the squeezing ellipse and therefore can be used as an error signal for the stabilization of the working point of the coherent control loop. To monitor residual phase noise, the second detection photo diode can be used as an out-of-loop sensor.

\section{Automatic alignment}

Relative misalignment between the squeezed light beam and the interferometer will reduce the effective squeezing level by inducing either optical losses (because of the reduced overlap of the squeezed field with the OMC and arm cavities) and phase noise, because of lock-point errors in the phase control [31]. The effect on phase noise is largely suppressed in our case by placing the phase control photo diode downstream of the OMC [32], and the dominant mechanism of squeezing degradation is through optical losses. A misalignment or displacement of the squeezed light beam in between the OPA and the asymmetric port of the interferometer reduces the power in the $\mathrm{TEM}_{00}$ mode and it is equivalent to a loss $L$ [31]:

$$
L \simeq\left(\frac{\tilde{\theta}_{x} \omega_{0} \pi}{\lambda}\right)^{2}+\left(\frac{\tilde{\Delta}_{x}}{\omega_{0}}\right)^{2}
$$

where $\omega_{0}$ is the radius of beam waist $(\sim 1 \mathrm{~mm}$ after the mode-matching telescope on the in-air bench), and $\tilde{\theta}_{x}$ and $\tilde{\Delta}_{x}$ are the rms values for the fluctuations in beam angle and displacement, respectively. The major contribution to alignment noise is from residual motion of the suspended detection bench hosting the telescope for mode matching between interferometer and OMC. This telescope has an angular magnification $\sim 20$ to match the large waist in the $3 \mathrm{~km}$ arm cavities with the small waist in the OMC. The residual angular motion of the suspended bench has an amplitude $\sim 1 \mu \mathrm{rad}$ around $0.5 \mathrm{~Hz}$ and therefore converts into $\sim 20 \mu \mathrm{rad}$ misalignment of the squeezed light beam. Long-term angular drifts of the suspended bench amount to several $\mu \mathrm{rad}$ and would produce comparably larger misalignment of the squeezed light beam. We apply a dualstage control to correct for angular misalignment by acting on the tip-tilt actuated steering mirrors on the in-air bench. A spurious polarization component of the beam reaching the detection bench from the interferometer side, representing a fraction $\sim 10^{-4}$ of the optical power, is reflected off the Faraday isolator towards the external squeezing bench. We detect that beam, after another reflection from a Faraday isolator on the in-air bench, with image sensors in near and far field configurations, respectively. The signals of these two sensors provide control signals to correct for misalignment which originates from detection bench motion. Such control signals have large dynamic range and allow us to track alignment changes even when squeezed light is not injected, i.e., during lock acquisition or commissioning of the interferometer. However, these signals do not provide information on a possible misalignment originating from the in-air bench. For a more accurate alignment sensing we directly probe the coupling of the squeezed light on the OMC. For this we apply small angular dithers to the squeezed light beam at four different frequencies below $20 \mathrm{~Hz}$ with the two steering mirrors along orthogonal axes. Demodulation of the beat note between the interferometer carrier and the coherent control field on the detection photodiodes at the dither frequencies yields alignment error signals to control the steering mirrors.

\section{MEASUREMENTS AND RESULTS}

Since the beginning of the $\mathrm{O} 3$ observation run, which started in April 2019, the Advanced Virgo detector sensitivity has routinely been improved via the injection of squeezed vacuum states of light. Measurements of spectral strain sensitivities demonstrating the effect of the squeezed light injection into the interferometer are shown in Fig. 2. The black trace illustrates a reference measurement, which is obtained when the interferometer is operated without squeezing. The squeezed light source is set to produce a squeezing level of approximately $10 \mathrm{~dB}$. By the injection of the squeezed vacuum field and proper tuning of the squeezing phase, a nonclassical strain sensitivity improvement of up to $3.2 \pm 0.1 \mathrm{~dB}$ can be observed (red trace). Shifting the squeezing phase by $90^{\circ}$ via the coherent control loop yields a measurement of the antisqueezing level, shown as the upper (blue) trace in Fig. 2. The antisqueezed interferometer strain sensitivity corresponds to an $8.5 \pm$ $0.1 \mathrm{~dB}$ increase in shot noise. Since only quantum noise can be manipulated by squeezing, any underlying nonquantum noise influences the level of the measured improvement. By subtracting the known technical noise in the Advanced Virgo detector (equivalent to $S_{t}=0$, see below) from both shot noise and squeezed noise measurements we infer a corresponding squeezing level of $3.8 \pm 0.1 \mathrm{~dB}$.

The duty cycle of squeezed light injection during the first 5 months of the $\mathrm{O} 3$ science run, i.e., the fraction of science 


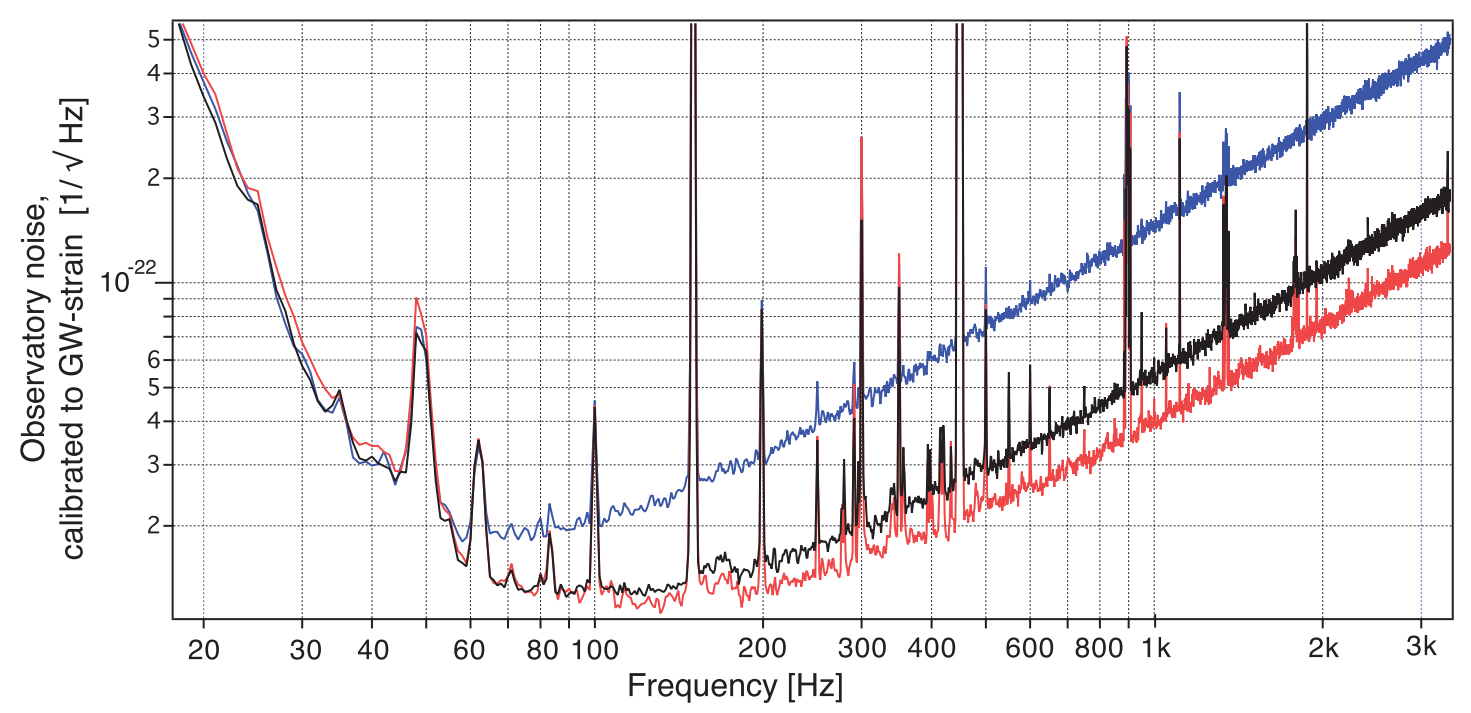

FIG. 2. Measured spectral strain sensitivity of the Advanced Virgo detector in different conditions of squeezed light injection. The black trace corresponds to the reference sensitivity in the absence of squeezed light. The measured sensitivity with squeezing or antisqueezing are shown as the red and blue traces, respectively. Our analysis yields a detected squeezing level of $3.2 \pm 0.1 \mathrm{~dB}$ with the corresponding antisqueezing of $8.5 \pm 0.1 \mathrm{~dB}$, normalized to the reference at $2.8 \mathrm{kHz}$. For this set of measurements, the injected squeezing level was about $10 \mathrm{~dB}$.

data acquired with squeezing enhanced detector sensitivity, is higher than $99 \%$. For routine operation during the science run, the injected squeezing level has been reduced to mitigate an enhanced radiation pressure effect due to the associated antisqueezing, which can be relevant at frequencies below $50 \mathrm{~Hz}$ [33]. Figure 3 shows the shot noise reduction measured over the period from April to September 2019. The sensitivity gain is measured as the ratio of highfrequency spectral strain sensitivity with and without squeezed light injection. However, during science runs, data without injected squeezing can only be acquired at the cost of a reduced performance of the GW observing network. Such data are thus only available for very short times during commissioning or calibration intervals. Slow fluctuations of

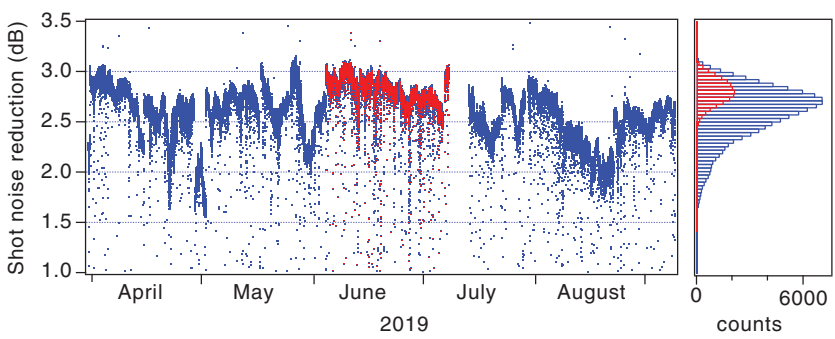

FIG. 3. Long-term performance of the shot noise reduction during the first 5 months of the $\mathrm{O} 3$ science run (1 April to 9 September 2019). Values are derived from the band limited rms strain sensitivity between 2650 and $3140 \mathrm{~Hz}$. The reference shot noise level in the absence of squeezing is calculated from the optical gain of the arm cavities (see text). Each point corresponds to an average over $100 \mathrm{~s}$ time. Data in red correspond to the time interval between 5 June and 8 July 2019. Data gaps are due to nonscience mode periods of the interferometer. Right: histogram of the collected data with bin width $0.036 \mathrm{~dB}$. the spectral strain sensitivity at high frequencies can be due to different reasons: changes in the amplitude of technical noise terms, changes in the shot noise level, or fluctuations in the effective squeezing level. In order to estimate the effective squeezing level, we determine the changes in shot noise level from the optical gain of the interferometer arm cavities, after an off-line calibration. Using available data without injected squeezing, we verified with a precision of $5 \%$ that the shot noise scales inversely with the optical gain, which is continuously measured using a permanent dither on arm cavity mirrors. The performance of the squeezing enhancement is dominated by instabilities of the interferometer, that lead to additional, nonstationary technical and optical loss contributions which so far cannot be compensated by control loops. The third month of data (marked in red in Fig. 3) corresponds to a period with stable conditions and as a result a rather constant shot noise reduction of $2.8 \pm 0.3 \mathrm{~dB}$ could be achieved.

A set of measurements of squeezed and antisqueezed sensitivities (such as Fig. 2) for different amounts of injected squeezing can be used to characterize the performance and limitations of the quantum noise reduction. Neglecting the radiation pressure contribution, the noise power spectral density $S_{p}$ of a quantum enhanced detector takes the form [34]

$$
S_{p}=S_{t}+S_{s n}\left\{1-4 \eta x\left[\frac{\left\langle\cos ^{2} \theta\right\rangle}{(1+x)^{2}}-\frac{\left\langle\sin ^{2} \theta\right\rangle}{(1-x)^{2}}\right]\right\},
$$

where $S_{s n}$ is the detector shot noise, $S_{t}$ contains the nonquantum noise contributions, $\eta$ is the squeezed beam optical path efficiency in terms of optical loss, $\theta$ is the rotation angle of the squeezing ellipse, and $x$ is the OPA nonlinear factor, 
which determines the level of produced squeezing. In general the angle of the squeezing ellipse $\theta$ (where $\theta=0$ and $\theta=\pi / 2$ correspond to squeezing and antisqueezing, respectively) can fluctuate around a mean value $\theta_{0}$ with a residual noise variance $\theta_{r m s}^{2}$. In this case the average operator \langle\rangle mixes the two terms in square brackets in Eq. (2) and as a consequence the potential sensitivity enhancement will be impaired by a fraction $\theta_{\mathrm{rms}}^{2}$ of antisqueezing. The effect of technical noise on the squeezing level is similar to having additional optical losses, and we can define an equivalent technical noise efficiency $\eta_{\text {tec }}=S_{s n} /\left(S_{s n}+S_{t}\right)$. The analysis of a series of measurements performed with different OPA-parametric amplification factors $x$ yields an overall efficiency $\eta_{\text {tot }}=\eta \eta_{\text {tec }}=59 \pm 2 \%$ and a residual phase noise $\theta_{\text {rms }}=45 \pm 40 \mathrm{mrad}$. As stated in Eq. (2) the effective squeezed light sensitivity enhancement depends on the interplay of the technical noise $S_{t}$, the optical losses, and the squeezing phase noise $\theta_{\mathrm{rms}}$. Here, the main contributions are discussed in the following subsections.

\section{A. Technical noise}

The electronic dark noise of the detection photodiodes is a relevant source of technical noise at high frequencies. With the current setup the dark noise clearance with respect to the shot noise is about $13 \mathrm{~dB}$. Additional contributions stem from residual laser frequency noise, fringe contrast defect, and amplitude noise of residual rf control sidebands. The laser frequency noise can couple into the output signal because asymmetries in the interferometer limit the common mode rejection factor. This contribution is reduced with an on-line subtraction by measuring the residual frequency noise from one of the interferometer control error signals and correcting the strain data after proper calibration. However, the efficiency of this frequency noise subtraction varies over time. The nonperfect fringe contrast contributes about $4 \%$ of the detected dc signal. Thus, the corresponding shot noise contribution cannot be affected by squeezing. Finally, about $0.4 \%$ of detected light is due to rf control sidebands leaking through the OMC, whose amplitude fluctuations contribute to technical noise. Considering all these contributions, the technical noise level is $9 \mathrm{~dB}$ below the unsqueezed shot noise $S_{s n}$ at $3 \mathrm{kHz}$. However, the actual amount of technical noise changes with time depending on the interferometer configuration.

\section{B. Optical losses}

Independent measurements were conducted to derive the optical loss budget. While some numbers can be measured with low uncertainty others are somewhat more difficult to access. The main sources of optical loss are listed in Table I, which also includes the equivalent technical noise efficiency $\eta_{\text {tec }}$. The overall optical losses $1-\eta_{\text {tot }}$ add up to $32 \%-41 \%$. We attribute the main uncertainties to nonstationary contributions of the technical noise and the
TABLE I. Estimated optical loss budget for the squeezing.

\begin{tabular}{lc}
\hline \hline Loss source & Loss $(\%)$ \\
\hline OPA escape efficiency & $1.0-1.5$ \\
Squeezed light source Faraday isolator & $1.6-2.0$ \\
Triple Faraday isolator on in-air bench & $2.5-3.0$ \\
Other optics on in-air bench & $0.5-1.5$ \\
Alignment jitter & $0.5-1.5$ \\
Mode mismatch to the interferometer & $3.0-5.0$ \\
Interferometer arm cavities & $3.0-5.0$ \\
Other interferometer optics & $2.0-6.0$ \\
Faraday isolator on detection bench (double-pass) & $1.5-2.5$ \\
Mode mismatch to OMC & $1.0-8.0$ \\
OMC intra-cavity losses & $1.5-2.5$ \\
OMC1 to OMC2 mismatch & $2.0-3.0$ \\
Detection control pick-off & $1.3-1.7$ \\
Efficiency of detection photodiodes & $0.7-1.3$ \\
Technical noise equivalents $\left(1-\eta_{\text {tec }}\right)$ & $7.0-15.0$ \\
Total & $\mathbf{3 2 - 4 1}$ \\
\hline \hline
\end{tabular}

mode-matching of the interferometer to the OMC. The latter is only experimentally measurable in the so-called "cold state" of the interferometer and thermal aberrations due to the interferometer being operated at full power ("hot state") can substantially change it.

\section{Squeezing phase noise}

The residual phase noise can be analyzed with the second detection photo diode as an out-of-loop sensor. With the current setup this allows for measurements with a bandwidth from dc $-25 \mathrm{kHz}$. After the engagement of the coherent control loop, the measured residual phase noise is approximately $20 \pm 5 \mathrm{mrad} \mathrm{rms}$. However, contributions at higher frequencies are not accessible with this measurement. About $8 \mathrm{mrad}$ rms additional phase noise is expected from the interferometer control sidebands at 8 and $56 \mathrm{MHz}$, which are partially transmitted by the OMCs [34]. Potential contributions of higher order modes to the phase noise budget are still under investigation. The above considerations show that most of the contributions to the optical loss and phase noise budget are understood while some contributions with their large uncertainties call for further analysis after the joint science run has ended.

\section{CONCLUSION}

The squeezing technology was successfully implemented in the Advanced Virgo detector and commissioned to enhance the detection sensitivity of the third joint LIGOVirgo observation run O3. Squeezed vacuum states of light are prepared on an external optical bench operated in air and are injected into the dark port of the Advanced Virgo interferometer. A shot noise reduction of up to $3.2 \pm 0.1 \mathrm{~dB}$ has been observed. The limiting factors have been analyzed and most are well understood. The findings presented here will be the basis for defining strategies to 
mitigate current limitations and move forward to an even more efficient quantum noise reduction. Via a reduction of technical noise contributions, the development of advanced mode matching strategies, and the implementation of a filter cavity which allows for a higher level of injected squeezing without disturbing the low frequencies (frequency-dependent squeezing), a broadband quantum noise reduction of 4-6 dB seems feasible with current technology. The squeezing injection was fully integrated and automatized to enable routine operation during the science run O3. Over the first 5 months, a duty cycle of more than 99\% was achieved. During this period several gravitationalwave candidates were observed $[35,36]$. In terms of overall detector sensitivity, typically characterized by the binary neutron star horizon, the squeezing injection leads to a $5 \%-8 \%$ improvement, which corresponds to $16 \%-26 \%$ increase of detection rate. The demonstrated improvement of the Advanced Virgo detector via the application of squeezed states of light underlines the importance of this quantum technology for ground-based gravitational-wave astronomy to further extend the observable volume of the Universe.

\section{ACKNOWLEDGMENTS}

The authors gratefully acknowledge the support of the Max Planck Society, Leibniz Universität Hannover, and Deutsche Forschungsgemeinschaft (DFG, German Research Foundation) through project Grant No. VA 1031/1-1 and under Germany's Excellence Strategy EXC 2123 QuantumFrontiers for the construction, installation, and operation of the squeezed light source. The authors gratefully acknowledge the Italian Istituto Nazionale di Fisica Nucleare (INFN), the French Centre National de la Recherche Scientifique (CNRS), and Netherlands Organization for Scientific Research, for the construction and operation of the Virgo detector and the creation and support of the EGO consortium. The authors also gratefully acknowledge research support from these agencies as well as by the Spanish Agencia Estatal de Investigación, the Conselleria d'Educació, Investigació, Cultura i Esport de la Generalitat Valenciana, the National Science Center of Poland, the Swiss National Science Foundation (SNSF), the European Commission, and the Hungarian Scientific Research Fund (OTKA). The authors gratefully acknowledge the support of the NSF, STFC, MPS, INFN, CNRS, and the State of Niedersachsen, Germany, for provision of computational resources.

[1] J. Aasi et al. (LIGO Scientific Collaboration), Classical Quantum Gravity 32, 074001 (2015).

[2] F. Acernese et al. (Virgo Collaboration), Classical Quantum Gravity 32, 024001 (2015).
[3] K. L. Dooley et al., Classical Quantum Gravity 33, 075009 (2016).

[4] V. B. Braginsky, Sov. Phys. JETP 26, 831 (1968).

[5] M. T. Jaekel and S. Reynaud, Europhys. Lett. 13, 301 (1990).

[6] C. M. Caves, Phys. Rev. D 23, 1693 (1981).

[7] M. Punturo et al., Classical Quantum Gravity 27, 084007 (2010).

[8] Y. Takeno, M. Yukawa, H. Yonezawa, and A. Furusawa, Opt. Express 15, 4321 (2007).

[9] H. Vahlbruch, M. Mehmet, K. Danzmann, and R. Schnabel, Phys. Rev. Lett. 117, 110801 (2016).

[10] R. E. Slusher, L. W. Hollberg, B. Yurke, J. C. Mertz, and J. F. Valley, Phys. Rev. Lett. 55, 2409 (1985).

[11] M. Mehmet and H. Vahlbruch, Classical Quantum Gravity 36, 015014 (2019).

[12] LIGO Scientific Collaboration, Nat. Phys. 7, 962 (2011).

[13] H. Grote, K. Danzmann, K. L. Dooley, R. Schnabel, J. Slutsky, and H. Vahlbruch, Phys. Rev. Lett. 110, 181101 (2013).

[14] AEI Hannover, research news, https://www.geo600 .org/1897380/gravitational-wave-detectors-begin-thirdobservation-run.

[15] J. Lough et al. (to be published).

[16] LIGO Scientific Collaboration, Nat. Photonics 7, 613 (2013).

[17] M. Tse et al., preceding Letter, Phys. Rev. Lett. 123, 231107 (2019).

[18] AdV Squeezing Working Group, Virgo-Technical Documentation System, Report No. VIR-0761B-17, 2017.

[19] B. Canuel, E. Genin, G. Vajente, and J. Marque Opt. Express 21, 10546 (2013).

[20] Virgo Collaboration, J. Instrum. 7, 3012 (2012).

[21] R. Bonnand, M. Ducrot, R. Gouaty, F. Marion, A. Masserot, B. Mours, E. Pacaud, L. Rolland, and M. Was, Classical Quantum Gravity 34, 175002 (2017).

[22] D. V. Martynov et al., Phys. Rev. A 95, 043831 (2017).

[23] Virgo Collaboration, Classical Quantum Gravity 32, 024001 (2015).

[24] W. P. Bowen, R. Schnabel, N. Treps, H.-A. Bachor, and P. K. Lam, J. Opt. B 4, 421 (2002).

[25] K. McKenzie, N. Grosse, W. P. Bowen, St. E. Whitcomb, M. B. Gray, D. E. McCelland, and P. K. Lam, Phys. Rev. Lett. 93, 161105 (2004).

[26] H. Vahlbruch, S. Chelkowski, K. Danzmann, and R. Schnabel, New J. Phys. 9, 371 (2007).

[27] H. Vahlbruch, S. Chelkowski, B. Hage, A. Franzen, K. Danzmann, and R. Schnabel, Phys. Rev. Lett. 97, 011101 (2006).

[28] E. Genin, M. Mantovani, G. Pillant, C. De Rossi, L. Pinard, C. Michel, M. Gosselin, and J. Casanueva, Appl. Opt. 57, 9705 (2018).

[29] S. Chua et al., Classical Quantum Gravity 31, 035017 (2014).

[30] F. Carbognani, Virgo-Technical Documentation System, Report No. VIR-0234C-17, 2017.

[31] E. Oelker, L. Barsotti, S. Dwyer, D. Sigg, and N. Mavalvala, Opt. Express 22, 21106 (2014).

[32] K. L. Dooley, E. Schreiber, H. Vahlbruch, C. Affeldt, J. R. Leong, H. Wittel, and H. Grote, Opt. Express 23, 8235 (2015). 
[33] H. Vahlbruch, M. Mehmet, H. Lück, K. Danzmann, and Virgo Collaboration (to be published).

[34] S. E. Dwyer, Ph. D. thesis, Massachusetts Institute of Technlogy, 2013.
[35] LIGO and Virgo Collaborations, https://gracedb.ligo.org/ latest/.

[36] LIGO and Virgo Collaborations, https://gcn.gsfc.nasa.gov/ gnc3/24168.gnc3.

F. Acernese,${ }^{1,2}$ M. Agathos,${ }^{3}$ L. Aiello, ${ }^{4,5}$ A. Allocca, ${ }^{6}$ A. Amato, ${ }^{7}$ S. Ansoldi,,${ }^{8,9}$ S. Antier, ${ }^{10}$ M. Arène,${ }^{10}$ N. Arnaud, ${ }^{11,12}$ S. Ascenzi, ${ }^{4,13}$ P. Astone, ${ }^{14}$ F. Aubin, ${ }^{15}$ S. Babak,${ }^{10}$ P. Bacon, ${ }^{10}$ F. Badaracco, ${ }^{4,5}$ M. K. M. Bader, ${ }^{16}$ J. Baird,${ }^{10}$ F. Baldaccini, ${ }^{17,18}$ G. Ballardin, ${ }^{12}$ G. Baltus, ${ }^{19}$ C. Barbieri, ${ }^{20,21}$ P. Barneo, ${ }^{22}$ F. Barone, ${ }^{23,2}$ M. Barsuglia, ${ }^{10}$ D. Barta ${ }^{24}$ A. Basti, ${ }^{25,6}$ M. Bawaj, ${ }^{26,18}$ M. Bazzan, ${ }^{27,28}$ M. Bejger, ${ }^{29}$ I. Belahcene, ${ }^{11}$ S. Bernuzzi, ${ }^{3}$ D. Bersanetti, ${ }^{30}$ A. Bertolini, ${ }^{16}$ M. Bischi, ${ }^{31,32}$ M. Bitossi, ${ }^{12,6}$ M. A. Bizouard, ${ }^{33}$ F. Bobba, ${ }^{34,35}$ M. Boer, ${ }^{33}$ G. Bogaert, ${ }^{33}$ F. Bondu, ${ }^{36}$ R. Bonnand, ${ }^{15}$ B. A. Boom, ${ }^{16}$ V. Boschi, ${ }^{6}$ Y. Bouffanais, ${ }^{27,28}$ A. Bozzi, ${ }^{12}$ C. Bradaschia, ${ }^{6}$ M. Branchesi,${ }^{4,5}$ M. Breschi,${ }^{3}$ T. Briant, ${ }^{37}$ F. Brighenti, ${ }^{31,32}$ A. Brillet, ${ }^{33}$ J. Brooks, ${ }^{12}$ G. Bruno, ${ }^{38}$ T. Bulik ${ }^{39}$ H. J. Bulten, ${ }^{40,16}$ D. Buskulic, ${ }^{15}$ G. Cagnoli ${ }^{7}$ E. Calloni, ${ }^{41,2}$ M. Canepa ${ }^{42,30}$ G. Carapella, ${ }^{34,35}$ F. Carbognani, ${ }^{12}$ G. Carullo, ${ }^{25,6}$ J. Casanueva Diaz, ${ }^{6}$ C. Casentini, ${ }^{43,13}$ J. Castañeda, ${ }^{22}$ S. Caudill, ${ }^{16}$ F. Cavalier, ${ }^{11}$ R. Cavalieri, ${ }^{12}$ G. Cella, ${ }^{6}$ P. Cerdá-Durán, ${ }^{44}$ E. Cesarini,${ }^{45,13}$ O. Chaibi,${ }^{33}$ E. Chassande-Mottin, ${ }^{10}$ F. Chiadini,${ }^{46,35}$ R. Chierici, ${ }^{47}$ A. Chincarini, ${ }^{30}$ A. Chiummo, ${ }^{12}$ N. Christensen, ${ }^{33}$ S. Chua ${ }^{37}$ G. Ciani, ${ }^{27,28}$ P. Ciecielag, ${ }^{29}$ M. Cieślar, ${ }^{29}$ R. Ciolfi, ${ }^{48,28}$ F. Cipriano, ${ }^{33}$ A. Cirone, ${ }^{42,30}$ S. Clesse, ${ }^{38}$ F. Cleva, ${ }^{33}$ E. Coccia,${ }^{4,5}$ P.-F. Cohadon, ${ }^{37}$ D. Cohen, ${ }^{11}$ M. Colpi ${ }^{20,21}$ L. Conti, ${ }^{28}$ I. Cordero-Carrión, ${ }^{49}$ S. Corezzi, ${ }^{17}, 18$ D. Corre, ${ }^{11}$ S. Cortese, ${ }^{12}$ J.-P. Coulon, ${ }^{33}$ M. Croquette,${ }^{37}$ J.-R. Cudell, ${ }^{19}$ E. Cuoco, ${ }^{12}$ M. Curylo, ${ }^{39}$ B. D'Angelo, ${ }^{42,30}$ S. D'Antonio, ${ }^{13}$ V. Dattilo, ${ }^{12}$ M. Davier, ${ }^{11}$ J. Degallaix, ${ }^{50}$ M. De Laurentis, ${ }^{41,2}$ S. Deléglise, ${ }^{37}$ W. Del Pozzo ${ }^{25,6}$ R. De Pietri, ${ }^{51,52}$ R. De Rosa ${ }^{41,2}$ C. De Rossi ${ }^{12}$ T. Dietrich, ${ }^{16}$ L. Di Fiore, ${ }^{2}$ C. Di Giorgio, ${ }^{34,35}$ F. Di Giovanni, ${ }^{44}$ M. Di Giovanni, ${ }^{53,54}$ T. Di Girolamo, ${ }^{41,2}$ A. Di Lieto, ${ }^{25,6}$ S. Di Pace,${ }^{55,14}$ I. Di Palma, ${ }^{55,14}$ F. Di Renzo, ${ }^{25,6}$ M. Drago, ${ }^{4,5}$ J.-G. Ducoin, ${ }^{11}$ O. Durante, ${ }^{34,35}$ D. D’Urso, ${ }^{56,57}$ M. Eisenmann, ${ }^{15}$ L. Errico, ${ }^{41,2}$ D. Estevez, ${ }^{15}$ V. Fafone,${ }^{43,13,4}$ S. Farinon ${ }^{30}$ F. Feng, ${ }^{10}$ E. Fenyvesi, ${ }^{24,58}$ I. Ferrante, ${ }^{25,6}$ F. Fidecaro, ${ }^{25,6}$ I. Fiori, ${ }^{12}$ D. Fiorucci, ${ }^{4,5}$ R. Fittipaldi, ${ }^{59,35}$ V. Fiumara,${ }^{60,35}$ R. Flaminio, ${ }^{15,61}$ J. A. Font, ${ }^{44,62}$ J.-D. Fournier, ${ }^{33}$ S. Frasca, ${ }^{55,14}$ F. Frasconi ${ }^{6}$ V. Frey, ${ }^{11}$ G. Fronzè ${ }^{63}$ F. Garufi, ${ }^{41,2}$ G. Gemme, ${ }^{30}$ E. Genin, ${ }^{12}$ A. Gennai, ${ }^{6}$ Archisman Ghosh, ${ }^{64,65,66,16}$ B. Giacomazzo,${ }^{53,54}$ M. Gosselin, ${ }^{12,25,6}$ R. Gouaty, ${ }^{15}$ A. Grado, ${ }^{67,2}$ M. Granata, ${ }^{50}$ G. Greco, ${ }^{31,32}$ G. Grignani, ${ }^{17,18}$ A. Grimaldi, ${ }^{53,54}$ S. J. Grimm, ${ }^{4,5}$ P. Gruning, ${ }^{11}$ G. M. Guidi, ${ }^{31,32}$ G. Guixé, ${ }^{22}$ Y. Guo, ${ }^{16}$ P. Gupta, ${ }^{16}$ O. Halim, ${ }^{5,4}$ T. Harder, ${ }^{33}$ J. Harms, ${ }^{4,5}$ A. Heidmann, ${ }^{37}$ H. Heitmann, ${ }^{33}$ P. Hello, ${ }^{11}$ G. Hemming, ${ }^{12}$ E. Hennes, ${ }^{16}$ T. Hinderer,${ }^{66,16,64}$ D. Hofman, ${ }^{50}$ D. Huet, ${ }^{11}$ V. Hui, ${ }^{15}$ B. Idzkowski, ${ }^{39}$ A. Iess, ${ }^{43,13}$ G. Intini, ${ }^{55,14}$ J.-M. Isac, ${ }^{37}$ T. Jacqmin, ${ }^{37}$ P. Jaranowski, ${ }^{68}$ R. J. G. Jonker, ${ }^{16}$ S. Katsanevas, ${ }^{12}$ F. Kéfélian, ${ }^{33}$ I. Khan, ${ }^{4,13}$ N. Khetan, ${ }^{4,5}$ G. Koekoek, ${ }^{16,69}$ S. Koley, ${ }^{16}$ A. Królak, ${ }^{70,71}$ A. Kutynia, ${ }^{70}$ D. Laghi, ${ }^{25,6}$ A. Lamberts, ${ }^{33,72}$ I. La Rosa, ${ }^{15}$ A. Lartaux-Vollard, ${ }^{11}$ C. Lazzaro, ${ }^{28}$ P. Leaci, ${ }^{55,14}$ N. Leroy, ${ }^{11}$ N. Letendre,${ }^{15}$ F. Linde ${ }^{73,16}$ M. Llorens-Monteagudo, ${ }^{44}$ A. Longo, ${ }^{74,75}$ M. Lorenzini, ${ }^{4,5}$ V. Loriette, ${ }^{76}$ G. Losurdo, ${ }^{6}$ D. Lumaca, ${ }^{43,13}$ A. Macquet, ${ }^{33}$ E. Majorana, ${ }^{14}$ I. Maksimovic, ${ }^{76}$ N. Man, ${ }^{33}$ V. Mangano,${ }^{55,14}$ M. Mantovani, ${ }^{12}$ M. Mapelli, ${ }^{27,28}$ F. Marchesoni,${ }^{26,18,77}$ F. Marion, ${ }^{15}$ A. Marquina, ${ }^{49}$ S. Marsat, ${ }^{10}$ F. Martelli, ${ }^{31,32}$ V. Martinez, ${ }^{7}$ A. Masserot, ${ }^{15}$ S. Mastrogiovanni, ${ }^{10}$ E. Mejuto Villa, ${ }^{78,35}$ L. Mereni, ${ }^{50}$ M. Merzougui, ${ }^{33}$ R. Metzdorff, ${ }^{37}$ A. Miani, ${ }^{53,54}$ C. Michel,,${ }^{50}$ L. Milano, ${ }^{41,2}$ A. Miller,,${ }^{55,14}$ E. Milotti, ${ }^{79,9}$ O. Minazzoli, ${ }^{33,80}$ Y. Minenkov, ${ }^{13}$ M. Montani, ${ }^{31,32}$ F. Morawski, ${ }^{29}$ B. Mours, ${ }^{81}$ F. Muciaccia,${ }^{55,14}$ A. Nagar, ${ }^{45,63,82}$ I. Nardecchia, ${ }^{43,13}$ L. Naticchioni, ${ }^{55,14}$ J. Neilson, ${ }^{78,35}$ G. Nelemans, ${ }^{83,16}$ C. Nguyen, ${ }^{10}$ D. Nichols, ${ }^{66,16}$ S. Nissanke,${ }^{66,16}$ F. Nocera, ${ }^{12}$ G. Oganesyan, ${ }^{4,5}$ C. Olivetto, ${ }^{12}$ G. Pagano, ${ }^{25,6}$ G. Pagliarolii, ${ }^{4,5}$ C. Palomba,${ }^{14}$ P. T. H. Pang, ${ }^{16}$ F. Pannarale ${ }^{55,14}$ F. Paoletti, ${ }^{6}$ A. Paoli, ${ }^{12}$ D. Pascucci, ${ }^{16}$ A. Pasqualetti, ${ }^{12}$ R. Passaquieti ${ }^{25,6}$ D. Passuello, ${ }^{6}$ B. Patricelli, ${ }^{25,6}$ A. Perego,${ }^{53,54}$ M. Pegoraro, ${ }^{28}$ C. Périgois, ${ }^{15}$ A. Perreca ${ }^{53,54}$ S. Perriès, ${ }^{47}$ K. S. Phukon, ${ }^{73,16}$ O. J. Piccinni, ${ }^{55,14}$ M. Pichot,${ }^{33}$ M. Piendibene ${ }^{25,6}$ F. Piergiovanni, ${ }^{31,32}$ V. Pierro, ${ }^{78,35}$ G. Pillant, ${ }^{12}$ L. Pinard,${ }^{50}$ I. M. Pinto, ${ }^{78,35,45}$ K. Piotrzkowski, ${ }^{38}$ W. Plastino, ${ }^{74,75}$ R. Poggiani, ${ }^{25,6}$ P. Popolizio, ${ }^{12}$ E. K. Porter,${ }^{10}$ M. Prevedelli, ${ }^{90}$ M. Principe,${ }^{78,45,35}$ G. A. Prodi, ${ }^{53,54}$ M. Punturo, ${ }^{18}$ P. Puppo, ${ }^{14}$ G. Raaijmakers, ${ }^{66,16}$ N. Radulesco,${ }^{33}$ P. Rapagnani,${ }^{55,14}$ M. Razzano, ${ }^{25,6}$ T. Regimbau, ${ }^{15}$ L. Rei, ${ }^{30}$ P. Rettegno, ${ }^{63,84}$ F. Ricci, ${ }^{55,14}$ G. Riemenschneider, ${ }^{84,63}$ F. Robinet, ${ }^{11}$ A. Rocchi, ${ }^{13}$ L. Rolland, ${ }^{15}$ M. Romanelli, ${ }^{36}$

R. Romano, ${ }^{1,2}$ D. Rosińska, ${ }^{39}$ P. Ruggi, ${ }^{12}$ O. S. Salafia,,${ }^{85,20,21}$ L. Salconi, ${ }^{12}$ A. Samajdar, ${ }^{16}$ N. Sanchis-Gual, ${ }^{86}$ E. Santos, ${ }^{33}$

B. Sassolas,${ }^{50}$ O. Sauter ${ }^{15}$ S. Sayah ${ }^{50}$ D. Sentenac, ${ }^{12}$ V. Sequino, ${ }^{30}$ A. Sharma,${ }^{4,5}$ M. Sieniawska, ${ }^{29}$ N. Singh,${ }^{39}$ A. Singhal, ${ }^{4,14}$ V. Sipala, ${ }^{56,57}$ V. Sordini, ${ }^{47}$ F. Sorrentino, ${ }^{30}$ M. Spera, ${ }^{27,28}$ C. Stachie, ${ }^{33}$ D. A. Steer, ${ }^{10}$ G. Stratta, ${ }^{87,32}$ A. Sur, ${ }^{29}$ B. L. Swinkels, ${ }^{16}$ M. Tacca, ${ }^{16}$ A. J. Tanasijczuk, ${ }^{38}$ E. N. Tapia San Martin, ${ }^{16}$ S. Tiwari, ${ }^{53,54}$ M. Tonelli, ${ }^{25,6}$ A. Torres-Forné, ${ }^{88}$ 
I. Tosta e Melo, ${ }^{56,57}$ F. Travasso, ${ }^{26,18}$ M. C. Tringali, ${ }^{39}$ A. Trovato, ${ }^{10}$ K. W. Tsang, ${ }^{16}$ M. Turconi, ${ }^{33}$ M. Valentini, ${ }^{53,54}$

N. van Bakel, ${ }^{16}$ M. van Beuzekom, ${ }^{16}$ J. F. J. van den Brand, ${ }^{40,69,16}$ C. Van Den Broeck, ${ }^{16,89}$ L. van der Schaaf, ${ }^{16}$ M. Vardaro, ${ }^{16,73}$ M. Vasúth, ${ }^{24}$ G. Vedovato,${ }^{28}$ D. Verkindt, ${ }^{15}$ F. Vetrano, ${ }^{31,32}$ A. Viceré, ${ }^{31,32}$ J.-Y. Vinet, ${ }^{33}$ H. Vocca, ${ }^{17,18}$ R. Walet, ${ }^{16}$ M. Was, ${ }^{15}$ A. Zadrożny, ${ }^{70}$ T. Zelenova, ${ }^{12}$ and J.-P. Zendri ${ }^{28}$

\title{
(Virgo Collaboration)
}

\author{
${ }^{1}$ Dipartimento di Farmacia, Università di Salerno, I-84084 Fisciano, Salerno, Italy \\ ${ }^{2}$ INFN, Sezione di Napoli, Complesso Universitario di Monte S.Angelo, I-80126 Napoli, Italy \\ ${ }^{3}$ Theoretisch-Physikalisches Institut, Friedrich-Schiller-Universität Jena, D-07743 Jena, Germany \\ ${ }^{4}$ Gran Sasso Science Institute (GSSI), I-67100 L'Aquila, Italy \\ ${ }^{5}$ INFN, Laboratori Nazionali del Gran Sasso, I-67100 Assergi, Italy \\ ${ }^{6}$ INFN, Sezione di Pisa, I-56127 Pisa, Italy \\ ${ }^{7}$ Université de Lyon, Université Claude Bernard Lyon 1, CNRS, Institut Lumière Matière, F-69622 Villeurbanne, France \\ ${ }^{8}$ Dipartimento di Matematica e Informatica, Università di Udine, I-33100 Udine, Italy \\ ${ }^{9}$ INFN, Sezione di Trieste, I-34127 Trieste, Italy \\ ${ }^{10}$ APC, AstroParticule et Cosmologie, Université Paris Diderot, \\ CNRS/IN2P3, CEA/Irfu, Observatoire de Paris, Sorbonne Paris Cité, F-75205 Paris Cedex 13, France \\ ${ }^{11}$ LAL, Univ. Paris-Sud, CNRS/IN2P3, Université Paris-Saclay, F-91898 Orsay, France \\ ${ }^{12}$ European Gravitational Observatory (EGO), I-56021 Cascina, Pisa, Italy \\ ${ }^{13}$ INFN, Sezione di Roma Tor Vergata, I-00133 Roma, Italy \\ ${ }^{14}$ INFN, Sezione di Roma, I-00185 Roma, Italy \\ ${ }^{15}$ Laboratoire d'Annecy de Physique des Particules (LAPP), Univ. Grenoble Alpes, Université Savoie Mont Blanc, \\ CNRS/IN2P3, F-74941 Annecy, France \\ ${ }^{16}$ Nikhef, Science Park 105, 1098 XG Amsterdam, The Netherlands \\ ${ }^{17}$ Università di Perugia, I-06123 Perugia, Italy \\ ${ }^{18}$ INFN, Sezione di Perugia, I-06123 Perugia, Italy \\ ${ }^{19}$ Université de Liège, B-4000 Liège, Belgium \\ ${ }^{20}$ Università degli Studi di Milano-Bicocca, I-20126 Milano, Italy \\ ${ }^{21}$ INFN, Sezione di Milano-Bicocca, I-20126 Milano, Italy \\ ${ }^{22}$ Departament de Física Quàntica i Astrofísica, Institut de Ciències del Cosmos (ICCUB), Universitat de Barcelona (IEEC-UB), \\ E-08028 Barcelona, Spain \\ ${ }^{23}$ Dipartimento di Medicina, Chirurgia e Odontoiatria "Scuola Medica Salernitana," Università di Salerno, \\ I-84081 Baronissi, Salerno, Italy \\ ${ }^{24}$ Wigner RCP, RMKI, H-1121 Budapest, Konkoly Thege Miklós út 29-33, Hungary \\ ${ }^{25}$ Università di Pisa, I-56127 Pisa, Italy \\ ${ }^{26}$ Università di Camerino, Dipartimento di Fisica, I-62032 Camerino, Italy \\ ${ }^{27}$ Università di Padova, Dipartimento di Fisica e Astronomia, I-35131 Padova, Italy \\ ${ }^{28}$ INFN, Sezione di Padova, I-35131 Padova, Italy \\ ${ }^{29}$ Nicolaus Copernicus Astronomical Center, Polish Academy of Sciences, 00-716, Warsaw, Poland \\ ${ }^{30}$ INFN, Sezione di Genova, I-16146 Genova, Italy \\ ${ }^{31}$ Università degli Studi di Urbino "Carlo Bo," I-61029 Urbino, Italy \\ ${ }^{32}$ INFN, Sezione di Firenze, I-50019 Sesto Fiorentino, Firenze, Italy \\ ${ }^{33}$ Artemis, Université Côte d'Azur, Observatoire Côte d'Azur, CNRS, CS 34229, F-06304 Nice Cedex 4, France \\ ${ }^{34}$ Dipartimento di Fisica “E.R. Caianiello,” Università di Salerno, I-84084 Fisciano, Salerno, Italy \\ ${ }^{35}$ INFN, Sezione di Napoli, Gruppo Collegato di Salerno, Complesso Universitario di Monte S. Angelo, I-80126 Napoli, Italy \\ ${ }^{36}$ Univ Rennes, CNRS, Institut FOTON-UMR6082, F-3500 Rennes, France \\ ${ }^{37}$ Laboratoire Kastler Brossel, Sorbonne Université, CNRS, ENS-Université PSL, Collège de France, F-75005 Paris, France \\ ${ }^{38}$ Université catholique de Louvain, B-1348 Louvain-la-Neuve, Belgium \\ ${ }^{39}$ Astronomical Observatory Warsaw University, 00-478 Warsaw, Poland \\ ${ }^{40}$ VU University Amsterdam, 1081 HV Amsterdam, The Netherlands \\ ${ }^{41}$ Università di Napoli “Federico II," Complesso Universitario di Monte S.Angelo, I-80126 Napoli, Italy \\ ${ }^{42}$ Dipartimento di Fisica, Università degli Studi di Genova, I-16146 Genova, Italy \\ ${ }^{43}$ Università di Roma Tor Vergata, I-00133 Roma, Italy \\ ${ }^{44}$ Departamento de Astronomía y Astrofísica, Universitat de València, E-46100 Burjassot, València, Spain \\ ${ }^{45}$ Museo Storico della Fisica e Centro Studi e Ricerche "Enrico Fermi," I-00184 Roma, Italy \\ ${ }^{46}$ Dipartimento di Ingegneria Industriale (DIIN), Università di Salerno, I-84084 Fisciano, Salerno, Italy \\ ${ }^{47}$ Institut de Physique des 2 Infinis de Lyon (IP2I)—UMR 5822, Université de Lyon, Université Claude Bernard,
}

CNRS, F-69622 Villeurbanne, France 


\footnotetext{
${ }^{48}$ INAF, Osservatorio Astronomico di Padova, I-35122 Padova, Italy

${ }^{49}$ Departamento de Matemáticas, Universitat de València, E-46100 Burjassot, València, Spain

${ }^{50}$ Laboratoire des Matériaux Avancés (LMA), IP2I-UMR 5822, CNRS, Université de Lyon, F-69622 Villeurbanne, France

${ }^{51}$ Dipartimento di Scienze Matematiche, Fisiche e Informatiche, Università di Parma, I-43124 Parma, Italy

${ }^{52}$ INFN, Sezione di Milano Bicocca, Gruppo Collegato di Parma, I-43124 Parma, Italy

${ }^{53}$ Università di Trento, Dipartimento di Fisica, I-38123 Povo, Trento, Italy

${ }^{54}$ INFN, Trento Institute for Fundamental Physics and Applications, I-38123 Povo, Trento, Italy

${ }^{55}$ Università di Roma "La Sapienza," I-00185 Roma, Italy

${ }^{56}$ Università degli Studi di Sassari, I-07100 Sassari, Italy

${ }^{57}$ INFN, Laboratori Nazionali del Sud, I-95125 Catania, Italy

${ }^{58}$ Institute for Nuclear Research (Atomki), Hungarian Academy of Sciences, Bem tér 18/c, H-4026 Debrecen, Hungary

${ }^{59}$ CNR-SPIN, clo Università di Salerno, I-84084 Fisciano, Salerno, Italy

${ }^{60}$ Scuola di Ingegneria, Università della Basilicata, I-85100 Potenza, Italy

${ }^{61}$ National Astronomical Observatory of Japan, 2-21-1 Osawa, Mitaka, Tokyo 181-8588, Japan

${ }^{62}$ Observatori Astronòmic, Universitat de València, E-46980 Paterna, València, Spain

${ }^{63}$ INFN Sezione di Torino, I-10125 Torino, Italy

${ }^{64}$ Delta Institute for Theoretical Physics, Science Park 904, 1090 GL Amsterdam, The Netherlands

${ }^{65}$ Lorentz Institute, Leiden University, PO Box 9506, Leiden 2300 RA, The Netherlands

${ }^{66}$ GRAPPA, Anton Pannekoek Institute for Astronomy and Institute for High-Energy Physics, University of Amsterdam, Science Park 904, 1098 XH Amsterdam, The Netherlands

${ }^{67}$ INAF, Osservatorio Astronomico di Capodimonte, I-80131 Napoli, Italy

${ }^{68}$ University of Biatystok, 15-424 Biatystok, Poland

${ }^{69}$ Maastricht University, P.O. Box 616, 6200 MD Maastricht, Netherlands

${ }^{70}$ NCBJ, 05-400 Świerk-Otwock, Poland

${ }^{71}$ Institute of Mathematics, Polish Academy of Sciences, 00656 Warsaw, Poland

${ }^{72}$ Lagrange, Université Côte d'Azur, Observatoire Côte d'Azur, CNRS, CS 34229, F-06304 Nice Cedex 4, France

${ }^{73}$ Institute for High-Energy Physics, University of Amsterdam, Science Park 904, 1098 XH Amsterdam, Netherlands

${ }^{74}$ Dipartimento di Matematica e Fisica, Università degli Studi Roma Tre, I-00146 Roma, Italy

${ }^{75}$ INFN, Sezione di Roma Tre, I-00146 Roma, Italy

${ }^{76}$ ESPCI, CNRS, F-75005 Paris, France

${ }^{77}$ Center for Phononics and Thermal Energy Science, School of Physics Science and Engineering, Tongji University, 200092 Shanghai, People's Republic of China

${ }^{78}$ Dipartimento di Ingegneria, Università del Sannio, I-82100 Benevento, Italy

${ }^{79}$ Dipartimento di Fisica, Università di Trieste, I-34127 Trieste, Italy

${ }^{80}$ Centre Scientifique de Monaco, 8 quai Antoine Ier, MC-98000, Monaco

${ }^{81}$ Université de Strasbourg, CNRS, IPHC UMR 7178, F-67000 Strasbourg, France

${ }^{82}$ Institut des Hautes Etudes Scientifiques, F-91440 Bures-sur-Yvette, France

${ }^{83}$ Department of Astrophysics/IMAPP, Radboud University Nijmegen, P.O. Box 9010, 6500 GL Nijmegen, The Netherlands

${ }^{84}$ Dipartimento di Fisica, Università degli Studi di Torino, I-10125 Torino, Italy

${ }^{85}$ INAF, Osservatorio Astronomico di Brera sede di Merate, I-23807 Merate, Lecco, Italy

${ }^{86}$ Centro de Astrofísica e Gravitação (CENTRA), Departamento de Física, Instituto Superior Técnico, Universidade de Lisboa, 1049-001 Lisboa, Portugal

${ }^{87}$ INAF, Osservatorio di Astrofisica e Scienza dello Spazio, I-40129 Bologna, Italy

${ }^{88}$ Max Planck Institute for Gravitationalphysik (Albert Einstein Institute), D-14476 Potsdam-Golm, Germany

${ }^{89}$ Department of Physics, Utrecht University, 3584CC Utrecht, Netherlands

${ }^{90}$ Department of Physics and Astronomy, Bologna University, I-40126 Bologna, Italy
} 